\title{
Transport of Very Low Density Lipoprotein Triglycerides in Varying Degrees of Obesity and Hypertriglyceridemia
}

\author{
Scott M. Grundy, Henry Y. I. Mok, Loren Zech, Daniel Steinberg, and \\ Mones Berman, Department of Medicine, Veterans Administration Hospital, and \\ University of California, San Diego, California 92093; and Laboratory of \\ Theoretical Biology, Division of Cancer, Biology and Diagnosis, National Cancer \\ Institute, National Institutes of Health, Bethesda, Maryland 20205
}

A B S T R A C T Measurements of transport of triglycerides (TG) in very low density lipoproteins (VLDL) were carried out in 59 patients by injection of radioactive glycerol, determinations of specific activities of VLDL-TG for $48 \mathrm{~h}$ thereafter, and treatment of the data by multicompartmental analysis. The patients were divided into three groups: normal weight (89-120\% ideal weight), mildly obese (120-135\% ideal weight), and markedly obese ( $135 \%$ ideal weight). They had varying levels of VLDL-TG ranging from normal to markedly elevated. In many subjects, there was a positive correlation between concentrations and transport of VLDL indicating that overproduction of VLDL-TG contributed to hypertriglyceridemia. In others, and particularly in several markedly obese subjects, transport rates were greatly increased without significant hypertriglyceridemia, suggesting that they had enhanced capacity to clear TG. In all groups, however, there were patients whose degree of hypertriglyceridemia seemed out of proportion to their transport rates. This finding and the fact that many patients have increased secretion of VLDL-TG without elevated plasma TG suggests that both overproduction of VLDL-TG and insufficient enhancement of clearance contributed to the development of hypertriglyceridemia.

The data showed a poor correlation between transport rates determined by our multicompartment analysis and single-exponential analysis used previously by other investigators $(r=0.46)$; this comparison was not improved by segregating patients according to their degree of obesity. Although two conversion pathways (fast and slow synthetic paths) were required to fit the data, there was no correlation between transport rates

This work was presented, in part, at the 49th and 50th Scientific Sessions of the American Heart Association, Miami Beach, Fla., November 1976 and 1977.

Received for publication 19 June 1978 and in revised form 26 January 1979. and the ratio of the two pathways. Also, despite the known pathway of conversion of VLDL to low density lipoprotein, no correlation was found between VLDL-TG transport rates and estimated low density lipoproteincholesterol concentrations.

\section{INTRODUCTION}

Elevations of very low density lipoproteins (VLDL) ${ }^{1}$ with hypertriglyceridemia are among the most common forms of hyperlipidemia. Underlying mechanisms for increased plasma VLDL are poorly understood. Some investigators have postulated that overproduction of VLDL is the predominant mechanism (1-5) while others have concluded that defective VLDL removal or degradation is of more importance (6-10). Most likely hypertriglyceridemia is heterogeneous in its pathogenesis, but at the moment there are uncertainties that preclude any final assessment. These uncertainties stem, in part, from differences in methods used by various investigators and from the limited number of subjects examined in most series. In this study, we have used a new approach to the quantification of the turnover of VLDL triglycerides (VLDL-TG) after injection of labeled glycerol, taking into account the complexity of the kinetics of the system, something not always recognized in previous studies. The development and validation of the multicompartmental analysis used in this study are described in the accompanying paper (11). Here we present results in a large group of subjects, 59 in all, with varying levels of plasma triglyceride (TG) and varying degrees of obesity. In this series studied under a common protocol and metabolic ward conditions, hypertriglyceridemia was commonly as-

\footnotetext{
${ }^{1}$ Abbreviations used in this paper: apoB, apoprotein B; FCR, fractional catabolic rate; HDL, high density lipoprotein; IDL, intermediate density lipoprotein; IW, ideal body weight; LDL, low density lipoprotein; TG, triglyceride; VLDL, very low density lipoprotein.
} 
sociated with overproduction of VLDL-TG. However, increased production was not always accompanied by hypertriglyceridemia. Several markedly obese, normolipidemic subjects showed significantly increased production but a comparable increase in removal (fractional catabolic rate). Finally, there was variability in capacity to clear VLDL-TG, indicating that a relative impairment in removal mechanisms augmented hypertriglyceridemia in some cases.

\section{METHODS}

Studies were carried out on the Special Diagnostic and Treatment Unit (metabolic unit), Veterans Administration Hospital, San Diego, Calif. Patients were divided into three categories: (a) normal weight (89-120\% of ideal weight), (b) mildly obese (121-135\% ideal weight), and (c) markedly obese ( $>136 \%$ of ideal weight). Each group included some normolipidemic patients and some patients with triglyceride levels as high as $300-800 \mathrm{mg} / \mathrm{dl}$. Several of the obese subjects had slight abnormalities in glucose tolerance but none had fasting hyperglycemia. None had liver or gastrointestinal disease. Some of the patients had a history of atherosclerotic disease (previous myocardial infarction or peripheral vascular disease), but none had had a recent infarct nor were any in congestive heart failure. None of the subjects had been on reducing diets or drugs affecting plasma lipids for at least 2 mo before the study. At time of study, none of our patients with fasting hypertriglyceridemia had fasting chylomicronemia (Type V hyperlipoproteinemia) or estimated concentrations of low density lipoprotein (LDL) cholesterol $>210 \mathrm{mg} / \mathrm{dl}$, a level indicative of Type IIB according to Fredrickson and Levy (12). A total of 59 patients was studied. Informed consent was obtained from each patient.

Patients were admitted at least $7 \mathrm{~d}$ before the study and put on measured weight-maintenance diets $(40 \%$ of calories as fat, mostly lard), to insure steady state at the time of study. These diets, before initiation of the test diet, consisted of solid foods, liquid formula, or a combination of the two (13). Fat was eliminated from the diet $36 \mathrm{~h}$ before study to eliminate contributions from dietary chylomicrons and their degradation products, but the carbohydrate and protein intake were continued as frequent small feedings (every $3 \mathrm{~h}$ ) of liquid formula. This technique, which was developed in our laboratory (14), has been employed recently by Reardon et al. (15) for measuring turnover of apoprotein $\mathrm{B}$ (apoB); it insures constant levels of VLDL-TG throughout the study. It might be noted that this approach to the steady state is different from that used in several previous studies that were carried out during fasting. As mentioned in the companion paper (11), a small rise in VLDL-TG during the 1st $12-18 \mathrm{~h}$ of formula feeding was sometimes noted; but thereafter, a steady state was achieved. In almost all cases, this rise was $<10 \%$ of the value at the beginning of the study, i.e., the value obtained after an overnight fast.

After intravenous injection of radioactive glycerol $\left(1,3-{ }^{14} \mathrm{C}\right.$ labeled or $2-{ }^{3} \mathrm{H}$-labeled, or both) plasma VLDL was isolated at time intervals and the specific activity of TG glycerol was determined (11). Calculation of plasma VLDL-TG transport was carried out by multicompartmental analysis according to the techniques described in detail in our companion paper (11). In these calculations, it was assumed that the entire "tail" of the specific activity curve was entirely due to the slow synthesis pathway. In the Results section, a comment will be made about effects of assuming that a portion of the tail is due to a slowly turning-over component of VLDL-TG (see companion paper [11]).

For determination of VLDL-TG transport, the mass of VLDL-TG in the plasma compartment was multiplied by the fractional catabolic rate (FCR) estimated by multicompartmental analysis. Plasma mass of VLDL-TG was calculated from VLDL-TG concentration and the estimated plasma volume. The method of calculation of plasma volume was essentially that described by Nikkila and Kekki (16) and employed the following equation: plasma volume (liters) $=$ (ideal weight [kilograms] $\times 0.045)+($ excess weight [kilograms] $\times 0.010)$. Ideal weight was calculated from life insurance tables (17). The factor 0.045 assumes that the plasma volume is equal to $4.5 \%$ of ideal body weight (18). Excess weight is equal to total body weight minus ideal weight and is assumed to represent mainly adipose tissue. The factor 0.010 assumes that plasma volume in excess adipose tissue is $1 \%$ of its mass: this value was derived from the extensive studies of Alexander et al. (19) on plasma volume in obese subjects.

Turnover of VLDL-TG also was estimated from the apparent log-linear phase of decline in specific activity of VLDL-TG after the peak of the curve as described by Farquhar et al. (20). The slope of the log-linear phase was estimated by eye, as done by previous workers, and might differ slightly in some cases from the maximal rate of decline as estimated by computer. For estimation of VLDL-TG turnover by this method, the mass of VLDL-TG in the plasma compartment was multiplied by the FCR derived from $t_{1 / 2}$ of the apparent log-linear decline in specific activity.

LDL-cholesterol was approximated by the following equation: LDL-cholesterol (milligram per deciliter) equals total cholesterol minus (VLDL-cholesterol plus high density lipoproteins [HDL]-cholesterol), where VLDL cholesterol was measured directly and HDL-cholesterol was estimated according to the equations developed by Myers et al. (21). Although these equations do not provide an exact estimate of HDL-cholesterol, values should be accurate enough for our purposes (Results). The value estimated for LDL-cholesterol would, in fact, include cholesterol in both LDL and intermediate density lipoprotein (IDL) fractions.

\section{RESULTS}

Complete numerical data for all subjects divided into three groups (normal weight, mildly obese, and markedly obese) including both normolipidemic and hyperlipidemic subjects, are on repository with the National Auxiliary Publication Service and they are available to the reader upon request. ${ }^{2}$

Because patients were of very different heights and weights, it was necessary to try to arrive at an appropriate method for normalization of the results so that a meaningful comparison among the various groups could be made. For this purpose, transport rates for VLDL-TG

${ }^{2}$ An Appendix has been deposited with the National Auxiliary Publications Service (NAPS) as NAPS document 03409. This information may be ordered from ASIS/NAPS, Microfiche Publications, P. O. Box 3513, Grand Central Station, New York 10017. Remit in advance, in U. S. funds, $\$ 3.00$ for microfiche copy, or for photocopy, $\$ 5.00$ up to 20 pages plus $25 \notin$ for additional pages. Outside the U.S. and Canada add postage of $\$ 3.00$ for photocopy and $\$ 1.00$ for microfiche. Checks should be made payable to Microfiche Publications. 
are expressed in absolute terms (milligrams per hour) and then normalized in three different ways: $(a)$ milligrams per hour per deciliter plasma volume, $(b)$ milligrams per hour per kilogram total body weight, and (c) milligrams per hour per kilogram ideal body weight (IW). In Figs. 1-3, the different modes of normalization are compared. A common method for normalizing VLDL-TG transport data is to express them as milligrams per hour per kilogram of total body weight. Fig. 1 compares data expressed in this way with absolute transport rates (milligrams per hour). The data for both the mildly and markedly obese subjects clearly fall below and to the right of the data for subjects of normal weight. Calculation of the flux rates per kilogram total body weight, thus, may be misleading. In obese subjects, it decreases outputs to inordinately low values, as compared to absolute outputs. In other words, dividing outputs by a large mass of adipose tissue could obscure real increases in production of VLDL-TG, a process presumably confined to the liver and intestine. Of course, the large mass of adipose tissue may indirectly play a role in increased VLDL production (e.g., by releasing excess FFA). If, however, the adipose tissue is not itself producing VLDL, then including its mass in the denominator will in effect cancel out such indirect effects. Thus, even when the adipose tissue is involved in the pathologic state with respect to VLDL production, its mass should not be used in the denominator.

Fig. 2 plots transport rates expressed as milligrams per hour per kilogram IW against absolute rates. The plot reveals a tight correlation across a wide range of transport rates for subjects of all degrees of obesity. Fig. 3 compares transports expressed as milligrams per hour per kilogram IW with that expressed as milligrams per hour per deciliter plasma volume. Again, there was a high degree of correspondence for these two modes of normalization for subjects of all weights. Thus, transports estimated by either of the latter two methods of

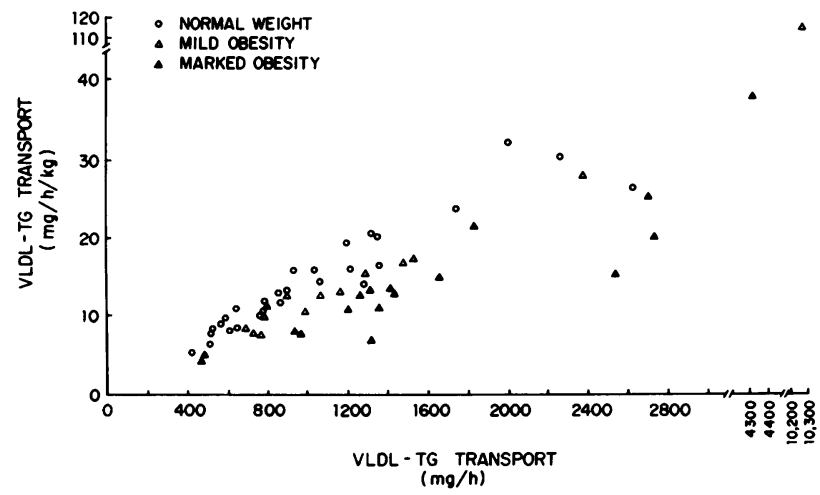

FIGURE 1 Comparison of VLDL-TG transport normalized per kilogram (milligrams per hour per kilogram) vs. uncorrected absolute values (milligrams per hour).

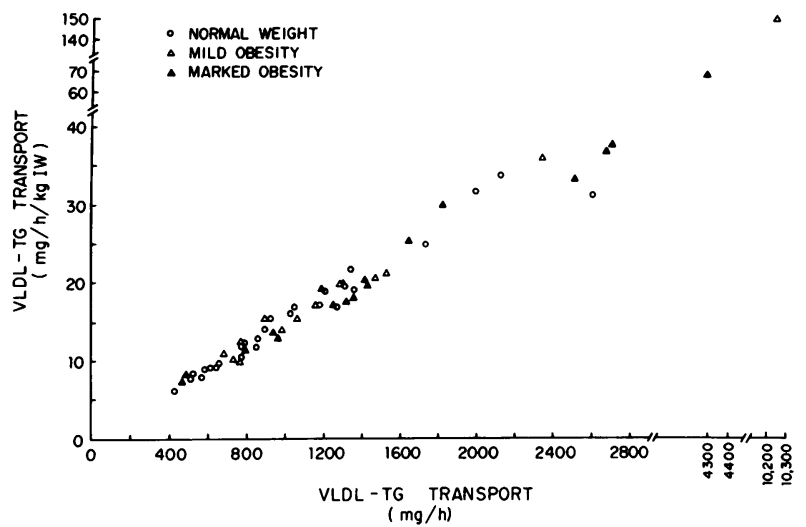

Figure 2 Comparison of VLDL-TG transport normalized per kilogram of ideal weight (milligrams per hour per kilogram IW) vs. uncorrected absolute values (milligrams per hour).

normalization would seem to be preferable to correction to kilograms of total body weight. In the subsequent presentation, the data will be normalized to either IW or deciliters of plasma volume when not expressed in absolute terms.

As indicated under Methods, the data presented in this section have not been corrected for the possible presence of a slowly turning-over component in plasma VLDL-TG. The companion paper (11, Eq. 7) suggests a procedure for estimating maximum contribution of this component. The calculation makes use of the cholesterol/TG ratio in VLDL. If it is assumed that this ratio for VLDL on the normal degradative pathway is 0.18 and that for a slower turning-over VLDL (e.g., $\beta$-VLDL) is 0.83 , the mass contribution of the latter can be estimated from the observed cholesterol/TG ratio. From Eq. 7 (11), the FCR for VLDL-TG can be corrected for the slow plasma component. This would yield a lower value for FCR than would be obtained by assuming that

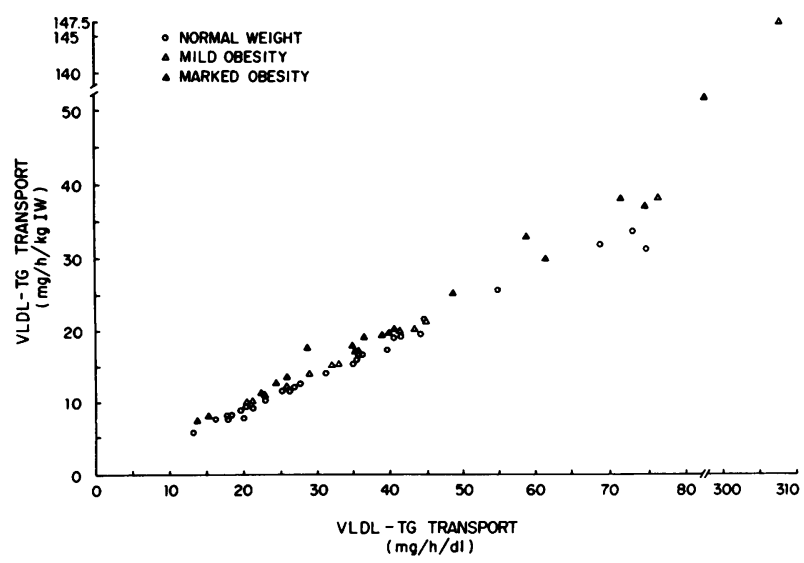

Figure 3 Comparison of VLDL-TG transport normalized per kilogram IW (milligrams per hour per kilogram IW) and to deciliter of plasma (milligrams per hour per deciliter). 
the tail is derived entirely from the slow synthesis pathway. This correction has been estimated for all studies, and the mean percentage of decrease in FCR for all 59 patients was $7 \pm 7 \%(\mathrm{SD})$. Because the correction was relatively small and because no trends were noted for any of the groups, it has not been applied to this data; i.e., the entire tail of the curve was assumed to have been caused by the slow synthesis pathway.

In Fig. 4, a comparison is made of VLDL-TG transport as determined by multicompartmental analysis (11) and by the method in which the initial slope after the peak is assumed to be first order (20). For all patients combined, the correlation between the two methods was poor $(r=0.45)$. In no single subcategory was the correlation good. It might be noted that the correlation was poor even for subjects of normal weight. However, the data obtained by these two methods of calculation did not show a completely random relationship and the correlation was, in fact, statistically significant $(P<0.05)$ for the whole group.

In Fig. 5, VLDL-TG concentrations in subjects of normal weight are plotted against transport rates. These data are compared with those obtained by Havel et al. (6) who made direct measurements of VLDL-TG production across the splanchnic bed using catheter techniques; their methods are free of many of the ambigui-

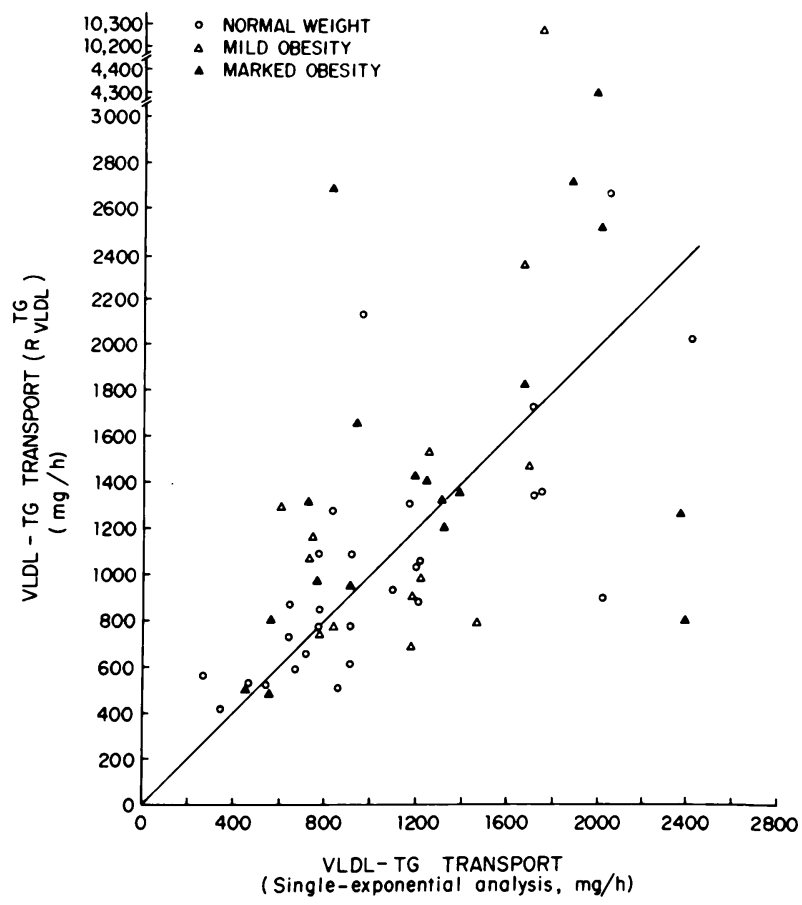

Figure 4 Comparison of VLDL-TG transport as determined by multicompartmental analysis and by single exponential analysis. The following correlation coefficients $(r)$ were noted: For normal weight subjects, $r=0.55$; for mildly obese, $r=0.50$; and for markedly obese, $r=0.60$. For the group as a whole, $r$ was 0.46 , and $P<0.05$.
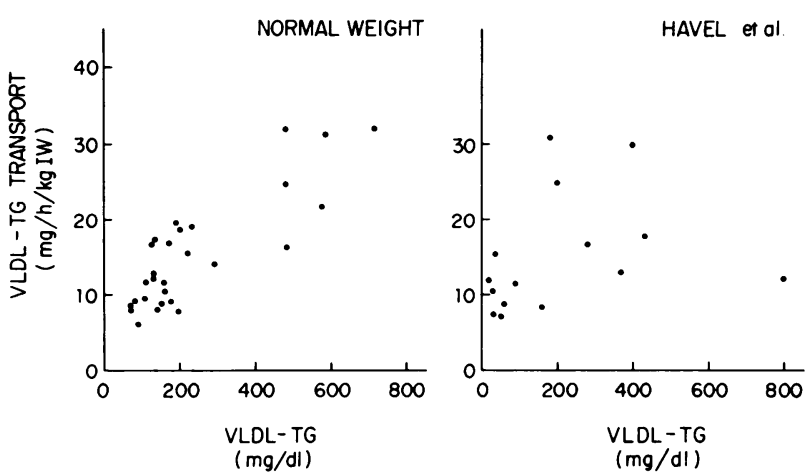

FIGURE 5 Transport rates vs. concentrations of VLDL-TG in subjects of normal weight (on the left). These data are compared with those of Havel et al. (6) (on the right) which were obtained by direct splanchnic measurements. For the data on the left, the correlation $(r)$ between transport rates and concentration was 0.85 .

ties associated with tracer methods. For most subjects with VLDL-TG concentrations in the normal range (i.e., in those with VLDL-TG $<200 \mathrm{mg} / \mathrm{dl}$ ), transport rates were similar in the two studies. In the study of Havel et al. (6), most patients with normal TG levels had production rates $<15 \mathrm{mg} / \mathrm{h}$ per $\mathrm{kg}$ IW. A comparable level of transport was noted in 14 of our 18 patients with VLDL-TG $<200 \mathrm{mg} / \mathrm{dl}$; the 4 remaining patients had higher transport rates as did the others with distinct hyperlipidemia. Examination of our data thus revealed that patients with hypertriglyceridemia generally had increased transport of VLDL-TG. However, it was not always greater than that in subjects who were able to maintain near normal VLDL-TG. In other words, at similar transport rates the FCR could be variable. Indeed, the FCR for our subjects of normal weight varied between 0.072 and $0.298 \mathrm{~h}^{-1}$. The relatively high FCR of several subjects with VLDL-TG production in the range of $15-20 \mathrm{mg} / \mathrm{h}$ per $\mathrm{kg}$ IW accounted for the fact that they had only modest hyperlipidemia. In the limited number of hypertriglyceridemic patients studied by Havel et al. (6), a variable pattern was noted. Although flux rates in hypertriglyceridemic patients were frequently greater than controls, this increase was not consistent in that some patients with increased plasma levels had outputs that were equal to or only slightly above those of control subjects. Thus, there was again considerable variability in FCR which contributed significantly to steady-state concentrations of VLDL-TG.

Plots of concentrations vs. transport are shown for patients with mild obesity and marked obesity in Fig. 6 . For subjects with mild obesity, the relation between these two parameters again was variable. Six subjects had VLDL-TG $<200 \mathrm{mg} / \mathrm{dl}$, and their transport rates were essentially normal (range $9.8-16.9 \mathrm{mg} / \mathrm{h}$ per $\mathrm{kg}$ IW). Two other patients with hypertriglyceridemia also 



Figure 6 Transport rates vs. concentrations of VLDL-TG in mildly obese and markedly obese subjects. Correlations $(r)$ between transport rates and concentrations were 0.94 and 0.17 , respectively. The former was statistically significant $(P<0.05)$, while the latter was not.

had relatively normal transport rates (10.7 and 12.3 $\mathrm{mg} / \mathrm{h}$ per $\mathrm{kg}$ IW), whereas in the others with increased VLDL-TG, transport was relatively high. One patient with mild obesity had a production rate $(147 \mathrm{mg} / \mathrm{h}$ per $\mathrm{kg} \mathrm{IW)} \mathrm{that} \mathrm{was} \mathrm{\cong 10-fold} \mathrm{normal,} \mathrm{while} \mathrm{his} \mathrm{VLDL-TG}$ concentration was increased in proportion (to 1,523 $\mathrm{mg} / \mathrm{dl})$. It is of interest to note that his FCR was maintained near the normal range, i.e., $0.202 \mathrm{~h}^{-1}$; thus, despite severe overproduction, this patient's removal mechanisms were not supersaturated.

In markedly obese subjects (Fig. 6 , right side), the relation between transport and concentration showed even greater variability. Several subjects had especially increased rates of transport with only mild or no increases in plasma levels; in the five most notable patients of this type, FCRs were unusually high, ranging from 0.278 to $0.737 \mathrm{~h}^{-1}$. Thus, despite their severe overproduction, their capacity to remove VLDL-TG was greatly enhanced, and clearly their removal capacity was not supersaturated. This phenomenon was not present in all the patients, however, and a few had VLDL-TG levels that were disproportionately high for their transport rates suggesting a clearance defect.

Fig. 7 examines the relation between VLDL-TG flux rates and the ratios of contribution of fast and slow input compartments (11). As secretion rates increase, there is the possibility that one pathway might become predominant. Indeed, in several patients with high transport rates, ratios of fast to slow paths were relatively low suggesting a preponderance of the slow pathway. However, with this exception, no correlation was noted between total flux and the ratio of fast to slow pathways in the remainder of the patients.

We also considered the possibility that the cholesterol: triglyceride ratio might be correlated with the rate

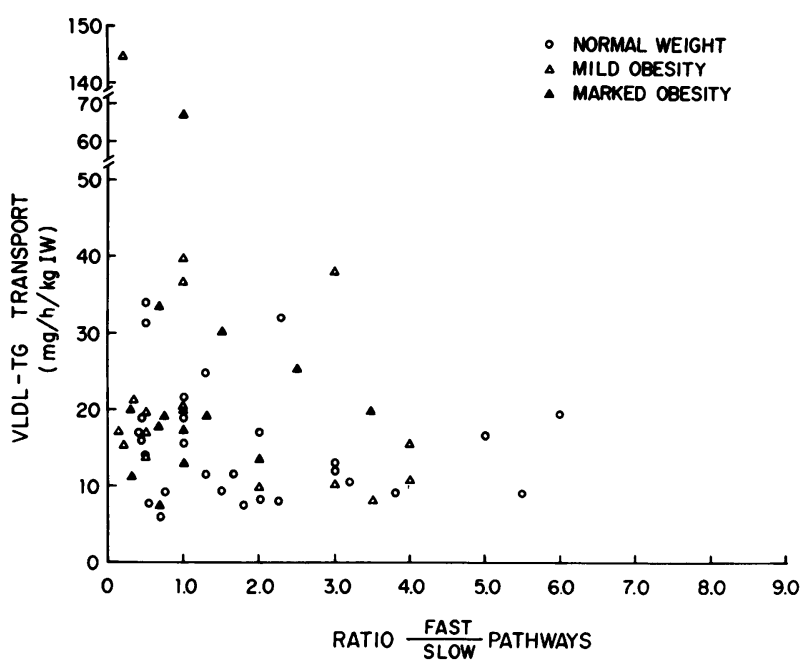

FIGURE 7 Transport of VLDL-TG plotted against the ratio of fast to slow pathways for synthesis form plasma glycerol. A significant correlation could not be demonstrated.

of influx of VLDL-TG. In other words, when secretion rates of VLDL-TG are high, each particle might be enriched with TG, thereby producing a low ratio of cholesterol to TG. The plot shown in Fig. 8, therefore, compares flux rates with cholesterol:triglyceride ratios. A weak correlation is present for subjects of normal weight which is in accord with the proposed notion; however, no correlation was found in the other groups, and clearly other factors also affect the ratio in circulating VLDL-TG.

It is generally thought that lipolysis of VLDL-TG causes production of progressively smaller lipoproteins



FigURE 8 Transport of VLDL-TG plotted against the ratio of cholesterol (CH) to triglycerides (TG) in VLDL. A significant correlation could not be demonstrated. 
with the end product of this degradative cascade being LDL. Therefore, an increased influx of VLDL might in turn lead to increased LDL. To examine this possibility, VLDL-TG transport was plotted against estimated LDL cholesterol, and no correlation was found between these two parameters for any single group or for all patients; in other words, increase influx of VLDL-TG was not necessarily associated with increased steadystate LDL-cholesterol levels.

\section{DISCUSSION}

Although both overproduction and defective removal of VLDL-TG may play a role in causation of hypertriglyceridemia, there has been continuing controversy as to which is most commonly responsible. In this study, multicompartmental analysis of VLDL-TG kinetics following injection of labeled glycerol was employed in an attempt to distinguish between these two mechanisms for hypertriglyceridemia and to determine which is predominant.

Farquhar et al. (20) introduced the use of radioactive glycerol as a precursor for investigation of VLDL-TG kinetics, and they have employed it in a series of studies on causation of hypertriglyceridemia $(1,2,22,23)$. Their studies were carried out for relatively short periods in fasting patients; specific activities of VLDL-TG were usually estimated for $12 \mathrm{~h}$ after injection of $\left[{ }^{3} \mathrm{H}\right]$ glycerol. In their work, they have assumed a caternary model for VLDL kinetics, i.e., a single pathway for glycerol incorporation into plasma VLDL and a homogenous VLDL-TG pool in plasma decaying in a simple, first-order fashion. To approximate this decay, they drew the best-fit, first-order slope through the points just after the initial peak of the specific activity curve of VLDL-TG. This approach has been adopted by several groups of investigators, and particularly by Nikkila and Kekki $(3,24,25)$.

In their original study, Farquhar et al. (20) observed that the decay curve for VLDL-TG specific activity did not always remain monoexponential but often showed a late flattening, a result not compatible with their proposed model. Nikkila and $\operatorname{Kekki}(3,24,25)$ also recognized that TG-specific activity curves were not consistently log-linear throughout the full extent of their decay (3), and the data that departed markedly from log-linear decay were excluded by these workers. They assumed that for the remaining patients single-exponential analysis should provide a reasonable approximation. It should be emphasized that in most of these previous studies, decay curves were followed for only $12-18 \mathrm{~h}$, whereas the departure from log-linear decay is often not readily apparent until later times.

Using single-exponential analysis, Farquhar et al. (1, $2,20,22,23$ ) have suggested that most instances of hypertriglyceridemia are due to increased influx of
VLDL-TG. Specifically, they concluded that concentrations of VLDL-TG are roughly proportional to influx rates up to a relatively fixed point of saturation of TG removal mechanisms. With any further increase in influx rates, plasma TG levels would increase very rapidly. According to this concept, therefore, VLDL production rate is the critical determinant of plasma TG concentrations. Nikkila and Kekki $(3,24,25)$, from their studies, have been in agreement that most cases in hypertriglyceridemia are associated with an overproduction of VLDL-TG. They have not concurred, however, with the postulate that plasma TG concentrations are determined almost solely by rates of TG influx but have suggested instead that removal rates are also an important factor. Although they contend that the relation between production and concentration of plasma TG adheres to saturation kinetics, they propose that saturation curves in different individuals exhibit considerable variability, i.e., that some people remove TG more effectively than others at the same production rates. According to this view, concentrations of plasma TG are regulated by the interaction between production and removal rates.

A somewhat different view of the pathogenesis of hypertriglyceridemia has been offered by Havel et al. (6) and Boberg et al. $(9,26)$. These workers performed direct measurements of splanchnic production of VLDL-TG in both normal and hypertriglyceridemic subjects. They found that many patients with elevated plasma TG did not have an enhanced influx of VLDL-TG and thus advanced the view that hypertriglyceridemia rarely originates from overproduction of VLDL-TG, but more often from defective degradation. Although the data of Havel et al. (6) disclosed that some of their patients with high TG had elevated secretion of VLDL-TG (Fig. 5), most others had VLDL-TG outputs in the normal range suggesting a removal defect. Taken as a whole, the data of Havel et al. (6) and Boberg et al. (9) have been interpreted as strong evidence for the predominance of faulty removal in the inducement of hypertriglyceridemia.

A similar but more tentative conclusion was drawn by Quarfordt et al. (27), who carried out multicompartmental analysis of specific activity curves of VLDL-TG after injection of a radioactive fatty acid. Using a multicompartmental model, which was a precursor to that employed in these investigations, these workers found little or no increase in the total transport of VLDL-TG in several patients with raised plasma TG. They, therefore, concluded that some patients can have defective removal of VLDL-TG. However, because the number of their patients was limited, care was taken not to generalize on the mechanism for increased TG in plasma.

This study represents an extension of the work of Farquhar et al. (20), in using labeled glycerol as a pre- 
cursor, and of Quarfordt et al. (27), in making use of multicompartmental analysis. The model used in this investigation should provide a better representation of true VLDL-TG kinetics than previous models because it is based on an expanded picture of the VLDL-TG decay curve and because it incorporates new information about lipoprotein and apoprotein kinetics that was previously unavailable. Moreover, our approach differs in another way from previous ones. To follow the specific activity curve for $48 \mathrm{~h}$ and yet maintain a steady state in VLDL-TG concentrations, a continuous input of dietary calories was required. Because most former studies were done during the fasting state over a shorter interval of time, differences in technique between ours and previous work might make a meaningful comparison tenuous. However, the fact that VLDL-TG transport in fasting normolipidemic subjects was similar to that found in fasting normolipidemic subjects using more direct methods, such as the studies of Havel et al. (6) (Fig. 5), suggests that transport rates under our conditions of study are not markedly different from those obtained after a relatively short period of fasting.

Our results indicate that markedly obese subjects were prone to increased transport of VLDL-TG compared to normolipidemic, normal-weight subjects. This overproduction is consistent with results of several prior studies in both man and animals (28-31). However, the importance of obesity as a cause of increased VLDL-TG flux may have been overlooked because of the method used for expressing results (32). A common method for expressing production rates is to divide absolute outputs by total body weight. As shown in Fig. 1, with this approach absolute production rates may be converted to misleadingly low values in obese subjects. Virtually all of the excess body weight in obesity resides in adipose tissue, which does not contribute to VLDL production, at least directly. Thus, if an obese patient and a nonobese subject who both had the same fat-free body weight and identical absolute production rates of VLDL-TG were compared per unit of total body weight, the obese subject would appear to have a lower secretion rate. By the same token, if transport per unit weight were found to be identical, this would actually correspond to greater absolute rates in the obese subjects. Therefore, we suggest that it would be preferable to express transport data per kilogram of IW which was shown to be closely correlated to absolute transport rates.

We might now explore the relations between enhanced VLDL-TG transport and elevated plasma concentrations. Several of our patients, particularly the markedly obese, had remarkably high transport rates (even exceeding those reported previously) and yet they had normal or only minimally elevated VLDL-
TG levels. A similar phenomenon has been recognized previously; for instance, Wolfe and Ahuja (33) infused glucose and fructose in hypercaloric amounts to human subjects and frequently found marked increases in splanchnic secretion of VLDL-TG without development of hypertriglyceridemia.

Thus, their patients and many of ours with overproduction of VLDL-TG seem to have been protected from hypertriglyceridemia because they could increase their clearance rates simultaneously. This observation implies that the relation between concentration and transport of VLDL-TG does not fit simple saturation kinetics, and certainly, a single saturation curve for removal does not apply to all patients. Presumably, only patients with faulty clearance or limited capacity to increase clearance will become hypertriglyceridemic when secretion of VLDL-TG is enhanced.

This is not to say that defective degradation of VLDL is necessarily the predominant mechanism by which hypertriglyceridemia develops. Most of our patients who had hypertriglyceridemia, whether normal or obese, also had an increased transport of VLDL-TG. Only an exceptional patient had appreciable elevation of plasma TG with a normal VLDLTG transport rate. Therefore, overproduction of VLDL-TG must contribute to hypertriglyceridemia in most patients. There was, nevertheless, considerable variability in capacity to remove TG from the circulation, which is to say the FCR was variable from patient to patient. Those with overproduction and rapid clearance were protected from hypertriglyceridemia while those with overproduction and a low FCR developed hypertriglyceridemia. With the exception of a few patients who had extreme overproduction or a severe defeat in removal, it appears that most patients with hypertriglyceridemia have two defects in TG metabolism: an absolute increase in VLDL-TG production and a relative defect in TG degradation. Whether a compensatory increase in TG removal in response to increased production is "normal" is not entirely clear. Support for this concept can be found in patients in our three groups who had relatively high VLDL-TG flux with normal plasma TG and in several others in the recent report of Wolfe and Ahuja (33) who failed to develop significantly elevated TG despite stimulation of VLDL-TG secretion with glucose or fructose infusion. Apparently, only patients with an inherently low FCR or an inability to adjust FCR will develop appreciably hypertriglyceridemia when challenged with an increased load of VLDL-TG. While in the final analysis it may be difficult to define what constitutes a "normal" or "abnormal" catabolic response to a given load of VLDL-TG, the general concept of the necessity for a combined defect for development of most cases of hypertriglyceridemia would appear valid. 
As yet, the basis for defective removal of VLDL-TG in patients with endogenous hypertriglyceridemia has not been determined. Although VLDL-TG is thought to be degraded by lipoprotein lipase, a deficiency of this enzyme has never been identified in patients with increases in VLDL without fasting chylomicronemia (34-36). We have shown that such patients can rapidly clear 10- to 20-fold increments in influx of TG when it is associated with chylomicrons (37). Delayed clearance of TG, therefore, seems to be limited largely to VLDL in these patients. This suggests that the basic abnormality may lie in the structure or composition of the VLDL particle itself, although no such abnormality has been demonstrated. An alternative explanation could be that lipoprotein lipase is faulty in its action upon VLDL and yet normal in its interaction with chylomicrons. The biochemical events leading to overproduction of VLDLTG also are understood poorly. In obesity, several factors could contribute to excess influx of VLDL-TG. First, ingestion of increased calories of any type, which are required for maintenance of the obese state, may offer the liver and gut an excess of substrate for synthesis of VLDL-TG (38). Second, FFA levels and fluxes are elevated in obesity, and these could provide another excess of precursor for VLDLTG (29, 39-42). Finally, insulin levels are often enhanced in obesity, and this has been proposed to stimulate VLDL-TG synthesis $(2,22,43-47)$. Thus, our finding of increased fluxes of VLDL in marked obesity could have several bases. On the other hand, the secretion of excess TG in patients of near normal weight is more difficult to explain. In this group, there were no obvious differences from those without hypertriglyceridemia, and while we must conclude that most of our hyperlipidemic subjects have a metabolic abnormality causing excess synthesis of VLDL-TG, the nature of this defect remains to be elucidated.

Because degradation of VLDL is thought to be the major source of $\operatorname{LDL}(48,49)$, patients with increased synthesis of VLDL might be expected to have elevated plasma LDL. Certainly, some patients with hyperlipidemia have increases in both VLDL and LDL (Type IIB hyperlipoproteinemia). However, we found no correlation between transport of VLDL-TG and concentrations of LDL. There are at least two possible explanations for this lack of correlation. First, excess synthesis of VLDL-TG need not always be associated with increased influx of VLDL-apoB. This possibility has been invoked by other workers $(50,51)$ including a preliminary report using the same methodology as employed in this work (52). Second, the "remnant" of VLDL may be removed by pathways other than conversion to LDL, particularly in hyperlipidemic subjects, as suggested for man by the work of Sigurdsson et al. (49), Reardon et al. (15), and Berman et al. (53), and for rats by Faergeman et al. (54).

\section{ACKNOWLEDGMENTS}

The authors wish to express their appreciation to Marjorie Whelan, Lianne Leipper, Joan Rupp and others of the nursing and dietetic services of the Special Diagnostic and Treatment Unit, and the Veterans Administration Hospital, San Diego, Calif., for their assistance on this project. Excellent technical assistance was provided by Elliott Groszek, Peter McGough, Susan Butler, Richard Earl, Warren May, and Avon Drummond.

This research was supported by the Medical Research Service of the Veterans Administration and by National Institutes of Health research grants HL-14197, awarded by the National Heart, Lung, and Blood Institute, and A.M16667 from the National Institute of Arthritis, Metabolism, and Digestive Disease U. S. Public Health Service/Department of Health, Education, and Welfare.

\section{REFERENCES}

1. Reaven, G. M., D. B. Hill, R. C. Gross, and J. W. Farquhar. 1965. Kinetics of triglyceride turnover of very low density lipoproteins of human plasma. J. Clin. Invest. 44: $1826-1833$.

2. Reaven, G., R. Lemer, M. Stern, and J. Farquhar. 1967. Role of insulin in endogenous hypertriglyceridemia. J. Clin. Invest. 46: 1756-1767.

3. Nikkila, E., and M. Kekki. 1971. Polymorphism of plasma triglyceride kinetics in normal human adult subjects. Acta. Med. Scand. 190: 49-59.

4. Nestel, P. J. 1966. Triglyceride turnover in coronary heart disease and the effect of dietary carbohydrate. Clin. Sci. (Oxf.). 31: 31-38.

5. Porte, D., Jr., and E. L. Bierman. 1969. The effect of heparin infusion on plasma triglyceride in vivo and in vitro with a method for calculating triglyceride turnover. J. Lab. Clin. Med. 73: 631-648.

6. Havel, R. J., J. P. Kane, E. O. Balasse, N. Segel, and L. V. Basso. 1970. Splanchnic metabolism of free fatty acids and production of triglycerides of very low density lipoproteins in normotriglyceridemic and hypertriglyceridemic humans. J. Clin. Invest. 49: 2017-2035.

7. Sailer, S., F. Sandhofer, and H. Braunsteiner. 1966. Umsatzraten fur freie Fettsauren und Triglyceride im Plasma bei essentieller Hyperlipamie. Klin. Wochenschr. 44: $1032-1036$.

8. Knittle, J. L., and E. H. Ahrens, Jr. 1964. Carbohydrate metabolism in two forms of hyperglyceridemia. J. Clin. Invest. 43: 485-495.

9. Boberg, J., L. Carlson, U. Freyschuss, B. Lessers, and M. Wahlquist. 1972. Splanchnic secretion rates of plasma triglycerides and total and splanchnic turnover of plasma free fatty acids in men with normo- and hypertriglyceridemia. Eur. J. Clin. Invest. 2: 454-466.

10. Ryan, W. G., and T. B. Schwartz. 1965. Dynamics of plasma triglyceride turnover in man. Metab. Clin. Exp. 14: $1243-1254$.

11. Zech, L. A., S. M. Grundy, D. Steinberg, and M. Berman. 1979. Kinetic model for production and metabolism of very low density lipoprotein triglycerides. Evidence for a slow production pathway and results for normolipidemic subjects. J. Clin. Invest. 63: 1262-1273.

12. Fredrickson, D. S., and R. I. Levy. 1972. Familial 
hyperlipoproteinemia. In The Metabolic Basis of Inherited Disease. J. B. Stanbury, J. B. Wyngaarden, and D. S. Fredrickson, editors. McGraw-Hill Book Company, New York. 3rd edition. 1: 545-614.

13. Grundy, S. M. 1975. Effects of polyunsaturated fats on lipid metabolism in patients with hypertriglyceridemia. J. Clin. Invest. 55: 269-282.

14. Grundy, S. M., P. J. Nestel, R. Monell, H. Mok, K. von Bergmann, and D. Steinberg. 1975. Kinetics of very low density lipoprotein-triglycerides (VLDL-TG) following radioglycerol. Circulation. 52(Suppl. II): 39.

15. Reardon, M. F., N. H. Fidge, and P. J. Nestel. 1978. Catabolism of very low density lipoprotein B apoprotein in man. J. Clin. Invest. 61: 850-860.

16. Nikkila, E. A., and M. Kekki. 1972. Plasma triglyceride metabolism in thyroid disease. J. Clin. Invest. 51: 21032114.

17. Metropolitan Life Insurance Company Statistical Bulletin. 1959. November, December. 40.

18. Edelman, I. S., and J. Liebman. 1959. Anatomy of body water and electrolytes. Am. J. Med. 27: 256-277.

19. Alexander, J. K., E. W. Dennis, W. G. Smith, K. H. Amad, W. C. Duncan, and R. C. Austin. 1962. Blood volume, cardiac output and distribution of systemic blood flow in extreme obesity. Cardiovasc. Res. Cent. Bull. (Houston). 1: 39.

20. Farquhar, J. W., R. C. Gross, R. M. Wagner, and G. M. Reaven. 1965. Validation of an incompletely coupled twocompartment nonrecycling catenary model for turnover of liver and plasma triglyceride in man. J. Lipid Res. 6: $119-134$.

21. Myers, L. H., N. R. Phillips, and R. J. Havel. 1976. Mathematical evaluation of methods for estimation of the concentration of the major lipid components of human serum lipoproteins. J. Lab. Clin. Med. 88: 491-505.

22. Farquhar, J. W., A. Frank, R. C. Gross, and G. M. Reaven. 1966. Glucose, insulin, and triglyceride response to high and low carbohydrate diets in man. J. Clin. Invest. 45: $1648-1656$.

23. Olefsky, J., J. W. Farquhar, and G. M. Reaven. 1974. Sex difference in the kinetics of triglyceride metabolism in normal and hypertriglyceridaemic human subjects. Eur. J. Clin. Invest. 4: 121-217.

24. Nikkila, E. A. 1969. Control of plasma and liver triglyceride kinetics by carbohydrate metabolism and insulin. Adv. Lipid Res. 7: 63-134.

25. Nikkila, E. A., and M. Kekki. 1972. Plasma endogenous triglyceride transport in hypertriglyceridaemic and effect of a hypolipidaemic drug (SU-13437). Eur. J. Clin. Invest. 2: 231-238.

26. Boberg, J., L. A. Carlson, and U. Freyschuss. 1972. Determination of splanchnic secretion rate of plasma triglycerides and of plasma free fatty acid total and splanchnic turnover. Eur. J. Clin. Invest. 2: 123-132.

27. Quarfordt, S. H., A. Frank, D. M. Shames, M. Berman, and D. Steinberg. 1970. Very low density lipoprotein triglyceride transport in type IV hyperlipoproteinemia and the effects of carbohydrate-rich diets. J. Clin. Invest. 49: 2281-2297.

28. Miller, H. I., W. M. Bortz, and B. C. Durham. 1968. The rate of appearance of plasma FFA in plasma triglyceride of normal and obese subjects. Metab. Clin. Exp. 17: 515-521.

29. Nestel, P. J., and H. M. Whyte. 1968. Plasma free fatty acid and triglyceride turnover in obesity. Metab. Clin. Exp. 17: 1122-1128.

30. Robertson, R. P., D. J. Gavareski, J. D. Henderson, D.
Porte, Jr., and E. L. Bierman. 1973. Accelerated triglyceride secretion: a metabolic consequence of obesity. J. Clin. Invest. 52: 1620-1626.

31. Nestel, P. J. 1973. Triglyceride turnover in man: effects of dietary carbohydrate. Prog. Biochem. Pharmacol. 8: 125-160.

32. Reaven, G. M., and R. M. Bernstein. 1978. Effects of obesity on the relationship between very low density lipoprotein production rate and plasma triglyceride concentration in normal and hypertriglyceridemic subjects. Metab. Clin. Exp. 27: 1047-1054.

33. Wolfe, B. M., and S. P. Ahuja. 1977. Effects of intravenously administered fructose and glucose on splanchnic secretion of plasma triglycerides in hypertriglyceridemic men. Metab. Clin. Exp. 26: 963-978.

34. Boberg, J., J. Augustin, M. L. Baginsky, P. Tejada, and W. V. Brown. 1977. Quantitative determination of hepatic and lipoprotein lipase activities from human posthepatin plasma. J. Lipid Res. 18: 544-547.

35. Nikkila, E. A., J. K. Huttunen, and C. Ehnholm. 1977. Effect of clofibrate on postheparin plasma triglyceride lipase activities in patients with hypertriglyceridemia. Metab. Clin. Exp. 26: 179-186.

36. Chait, A., W. R. Hazzard, J. J. Albers, R. P. Kushwaha, and J. D. Brunzell. 1978. Impaired very low density lipoprotein and triglyceride removal in broad beta disease: comparison with endogenous hypertriglyceridemia. Metab. Clin. Exp. 27: 1055-1066.

37. Grundy, S. M., and H. Y. I. Mok. 1976. Chylomicron clearance in normal and hyperlipidemic man. Metab. Clin. Exp. 25: 1225-1239.

38. Barter, P. J., and P. J. Nestel. 1972. Plasma free fatty acid transport during prolonged glucose consumption and its relationship to plasma triglyceride fatty acids in man. J. Lipid Res. 13: 483-490.

39. Dole, V. P. 1956. The relation between nonesterified fatty acids in plasma and the metabolism of glucose. J. Clin. Invest. 35: 150-154.

40. Gordon, E. S. 1960. Nonesterified fatty acids in blood of obese and lean subjects. Am. J. Clin. Nutr. 8: 740-747.

41. Opie, L. H., and P. G. Walfish. 1963. Plasma free fatty acid concentration in obesity. N. Engl. J. Med. 268: 757-760.

42. Corvilain, J., H. Loeb, A. Champenois, and M. Abramow. 1961. Effect of fasting on levels of plasma nonesterified fatty acids in normal children, normal adults, and obese adults. Lancet. I: 534-535.

43. Topping, D. L., and P. A. Mayes. 1972. The immediate effects of insulin and fructose on the metabolism of the perfused liver. Changes in lipoprotein secretion, fatty acid oxidation and esterification, lipogenesis and carbohydrate metabolism. Biochem. J. 126: 295-311.

44. Letarte, J., and T. Russell Fraser. 1969. Stimulation by insulin of the incorporation of ${ }^{14} \mathrm{C}$-U-glucose into lipids released by the liver. Diabetologia. 5: 358-359.

45. Haft, E. E. 1967. Effects of insulin on glucose metabolism by the perfused normal rat liver. Am. J. Physiol. 213: 219-230.

46. Ford, S., Jr., R. C. Bozian, and H. C. Knowles, Jr. 1968. Interactions of obesity and glucose and insulin levels in hypertriglyceridemia. Am. J. Clin. Nutr. 21: 904-910.

47. Bierman, E. L., and D. P. Porte, Jr. 1968. Carbohydrate intolerance and lipemia. Ann. Intern. Med. 68: 926-933.

48. Eisenberg, S., D. W. Bilheimer, R. I. Levy, and F. I. Lindgren. 1973. On the metabolic conversion of human 
plasma very low density lipoprotein to low density lipoprotein. Biochim. Biophys. Acta. 326: 361-377.

49. Sigurdsson, G., A. Nicoll, and B. Lewis. 1975. Conversion of very low density lipoprotein to low density lipoprotein. A metabolic study of apolipoprotein B kinetics in human subjects. J. Clin. Invest. 56: 1481-1490.

50. Ruderman, N. B., A. L. Jones, R. M. Krauss, and E. Shafrir. 1971. A biochemical and morphologic study of very low density lipoproteins in carbohydrate-induced hypertriglyceridemia. J. Clin. Invest. 50: 1355-1368.

51. Windmueller, H. G., and A. E. Spaeth. 1967. De novo synthesis of fatty acid in perfused rat liver as a determinant of plasma lipoprotein production. Arch. Biochem. Biophys. 122: 363.
52. Melish, J., A. L. Ngoc, H. Ginsberg, W. V. Brown, and D. Steinberg. 1977. Effect of high carbohydrate diet on very low density lipoprotein apoprotein-B and triglyceride production Circulation. 55(Suppl. III): 56. (Abstr.)

53. Berman, M., M. Hall, III, R. I. Levy, S. Eisenberg, D. W. Bilheimer, R. D. Phair, and R. H. Goebel. 1978. Metabolism of apoB and apoC lipoproteins in man: kinetic studies in normal and hyperlipoproteinemic subjects. J. Lipid Res. 19: 38-56.

54. Faergeman, O., S. Tsunako, J. P. Kane, and R. J. Havel. 1975. Metabolism of apoprotein B of plasma very low density lipoproteins in the rat. J. Clin. Invest. 56: 1396-1403. 